\title{
Rapid Response Systems Reduce In-Hospital Cardiopulmonary Arrest: A Pilot Study and Motivation for a Nationwide Survey
}

\author{
Yeonhee Park ${ }^{1}$, Jong-Joon Ahn², Byung Ju Kang ${ }^{2}$, Young Seok Lee ${ }^{3}$, Sang-Ook Ha ${ }^{4}$, Jin-Soo Min ${ }^{5}$, Woo-Hyun Cho ${ }^{6}$, \\ Se-Hee $\mathrm{Na}^{7}$, Dong-Hyun Lee ${ }^{8}$, Seung-Yong Park ${ }^{9}$, Goo-Hyeon Hong ${ }^{10}$, Hyun-Jung Kim ${ }^{11}$, Sangwoo Shim ${ }^{12}$, \\ Jung-Hyun Kim ${ }^{13}$, Seok-Jeong Lee ${ }^{14}$, So-Young Park ${ }^{15}$, and Jae Young Moon ${ }^{1}$
}

\begin{abstract}
${ }^{1}$ Division of Pulmonary and Critical Care Medicine, Department of Internal Medicine, Chungnam National University Hospital, Chungnam National University College of Medicine, Daejeon; ${ }^{2}$ Division of Pulmonary and Critical Care Medicine, Department of Internal Medicine, Ulsan University Hospital, University of Ulsan College of Medicine, Ulsan; ${ }^{3}$ Division of Respiratory and Critical Care Medicine, Department of Internal Medicine, Korea University Guro Hospital, Seoul; ${ }^{4}$ Department of Emergency Medicine, Hallym University Medical Center, Hallym University Sacred Heart Hospital, Anyang; ${ }^{5}$ Division of Pulmonology, Department of Internal Medicine, Chungbuk National University Hospital, Cheongju; ${ }^{6}$ Division of Pulmonary and Critical Care Medicine, Department of Internal Medicine, Pusan National University Yangsan Hospital, Yangsan; 'Department of Anesthesiology and Pain Medicine, Gangnam Severance Hospital, Yonsei University College of Medicine, Seoul; ${ }^{8}$ Division of Pulmonary and Critical Care Medicine, Department of Internal Medicine, Dong-A University College of Medicine, Busan; ${ }^{9}$ Division of Pulmonology and Allergy, Department of Internal Medicine, Chonbuk National University Hospital, Jeonju; ${ }^{10}$ Division of Pulmonology and Allergy, Department of Internal Medicine, Dankook University Hospital, Dankook University College of Medicine, Cheonan; ${ }^{11}$ Division of Pulmonary and Critical Care Medicine, Department of Internal Medicine, Kyungpook National University Hospital, Daegu; ${ }^{2}$ Department of Internal Medicine, Catholic University of Daegu School of Medicine, Daegu; ${ }^{13}$ Division of Pulmonology, Department of Internal Medicine, CHA Bundang Medical Center, CHA University, Seongnam; ${ }^{14}$ Department of Internal Medicine, Yonsei University Wonju College of Medicine, Wonju; ${ }^{15}$ Division of Pulmonary and Critical Care Medicine, Department of Internal Medicine, Kyung Hee University School of Medicine, Seoul, Korea
\end{abstract}

Background: Early recognition of the signs and symptoms of clinical deterioration could diminish the incidence of cardiopulmonary arrest. The present study investigates outcomes with respect to cardiopulmonary arrest rates in institutions with and without rapid response systems (RRSs) and the current level of cardiopulmonary arrest rate in tertiary hospitals.

Methods: This was a retrospective study based on data from 14 tertiary hospitals. Cardiopulmonary resuscitation (CPR) rate reports were obtained from each hospital to include the number of cardiopulmonary arrest events in adult patients in the general ward, the annual adult admission statistics, and the structure of the RRS if present.

Results: Hospitals with RRSs showed a statistically significant reduction of the CPR rate between 2013 and 2015 (odds ratio [OR], 0.731 ; $95 \%$ confidence interval [Cl], 0.577 to $0.927 ; P=0.009$ ). Nevertheless, CPR rates of 2013 and 2015 did not change in hospitals without RRS (OR, $0.988 ; 95 \% \mathrm{Cl}, 0.868$ to $1.124 ; \mathrm{P}=0.854)$. National university-affiliated hospitals showed less cardiopulmonary arrest rate than private university-affiliated in 2015 ( 1.92 vs. 2.40; OR, 0.800; 95\% Cl, 0.702 to 0.912; P = 0.001). High-volume hospitals showed lower cardiopulmonary arrest rates compared with medium-volume hospitals in 2013 (1.76 vs. 2.63; OR, 0.667; $95 \% \mathrm{Cl}, 0.577$ to $0.772 ; \mathrm{P}<$ 0.001 ) and in 2015 ( 1.55 vs. $3.20 ; \mathrm{OR}, 0.485 ; 95 \% \mathrm{Cl}, 0.428$ to $0.550 ; \mathrm{P}<0.001$ ).

Conclusions: RRSs may be a feasible option to reduce the CPR rate. The discrepancy in cardiopulmonary arrest rates suggests further research should include a nationwide survey to tease out factors involved in in-hospital cardiopulmonary arrest and differences in outcomes based on hospital characteristics.

Key Words: in-hospital cardiopulmonary arrest; patient safety; quality of health care; rapid response systems.

Received on January 13, 2017 Revised on May 3, 2017 Accepted on May 30, 2017

Correspondence to: Jae Young Moon, Division of Pulmonary and Critical Care Medicine, Department of Internal Medicine, Chungnam National University Hospital, Chungnam National University College of Medicine, 282 Munhwa-ro, Jung-gu, Daejeon 35015, Korea

Tel: +82-42-280-7870, Fax: +82-42-280-7877, E-mail: jymoon@cnuh.co.kr

*No potential conflict of interest relevant to this article was reported.

(c) This is an Open Access article distributed under the terms of the Creative Commons Attribution Non-Commercial License (http://creativecommons.org/ licenses/by-nc/4.0/) which permits unrestricted non-commercial use, distribution, and reproduction in any medium, provided the original work is properly cited. Copyright (c) 2017 The Korean Society of Critical Care Medicine 


\section{Introduction}

Patient safety and healthcare quality outcomes have been areas of increasing interest in Korea. In-hospital cardiopulmonary arrest is a known major patient safety and healthcare quality indicator. In most cases, clinical deterioration commonly precedes cardiopulmonary arrest [1-6]. Accordingly, early recognition of the signs and symptoms of deterioration could reduce the incidence of cardiopulmonary arrest, and this is the basis of rapid response systems (RRSs) [7,8].

In Korea, RRS implementation has been targeted at large academic medical centers. Although there have been some single-center studies, no nationwide survey has investigated the effect of RRSs on in-hospital cardiopulmonary arrest in Korea. Therefore, it is still necessary to estimate the effect of RRSs on cardiopulmonary arrest in domestic hospitals.

Two general hospitals in Korea implemented RRSs in 2014. The present study aimed to compare in-hospital cardiopulmonary arrest rates of institutions with or with- out RRSs, as well as to determine the cardiopulmonary arrest rates both before and after RRS implementation. We also used a pilot study to ascertain the prevalence of in-hospital cardiopulmonary arrest at tertiary hospitals, which was followed with a nationwide survey.

\section{Materials and Methods}

\section{1) Study design and institution selection}

This was a retrospective, multi-center study that used in-hospital cardiopulmonary arrest data from 14 tertiary hospitals from January 2013 to December 2015. Among the existing 43 tertiary hospitals nationwide, the study included data from two institutions with RRSs and 12 facilities without RRSs. We excluded two hospitals with more than 1,500 beds and one hospital with less than 700 beds. We also excluded five hospitals that had introduced an RRS before January 2013 and two hospitals that implemented an RRS after January 2015 (Figure 1).

From the remaining 33 hospitals, we selected 16 medi-

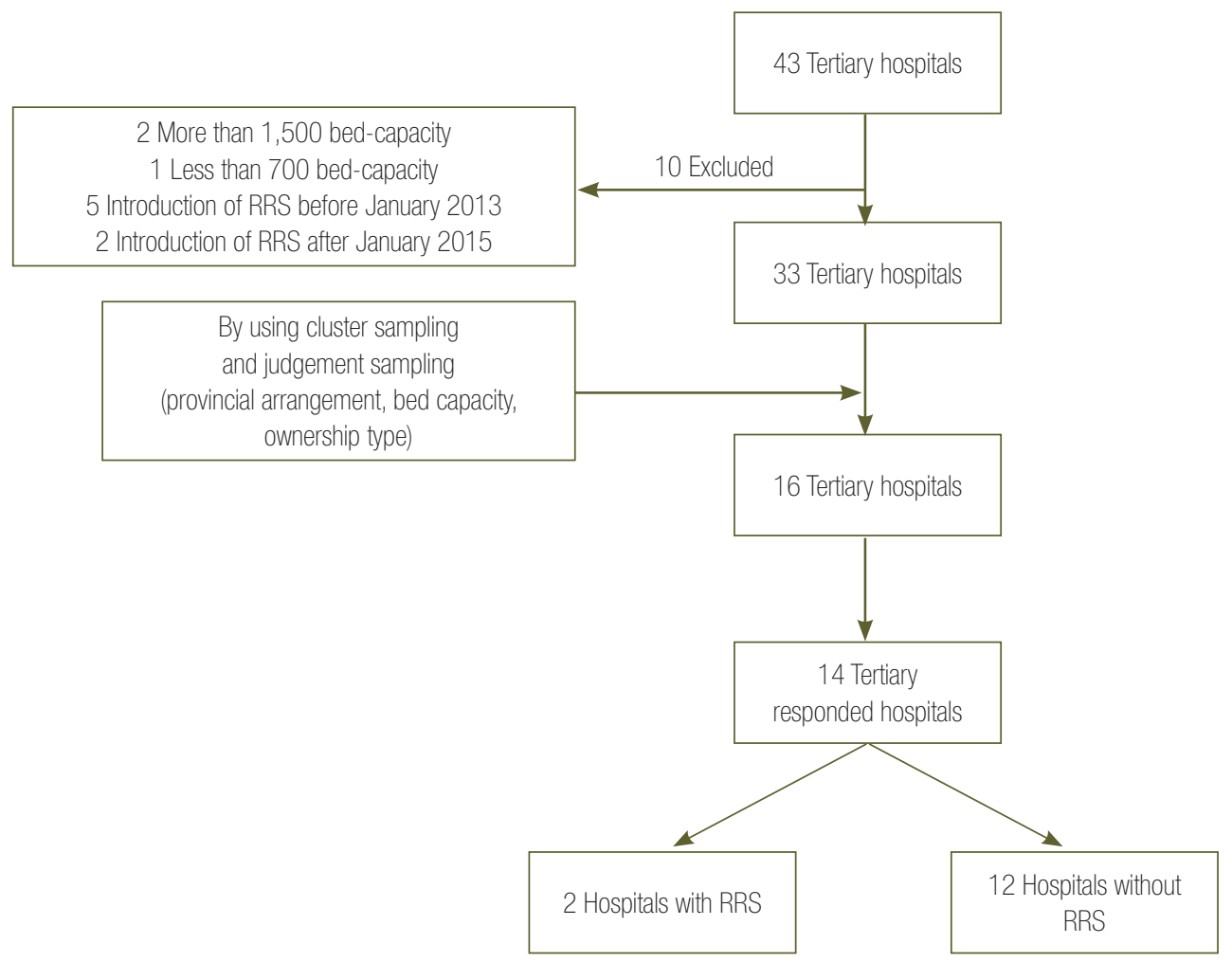

Figure 1. Selection process of included tertiary hospitals. RRS: rapid response system. 
cal centers in consideration of their provincial arrangement, bed capacity, and ownership type using cluster sampling and judgment sampling. These methods are appropriate when the sample size is relatively small. The population of all tertiary hospitals was believed to have reached or exceeded a certain level of healthcare quality. Of the 16 selected medical centers, 14 directors or physicians in charge of intensive care units were willing to participate in this study.

To identify the current in-hospital cardiopulmonary arrest rate in tertiary hospitals in Korea, we estimated the cardiopulmonary arrest rate of hospitals with RRSs and also those without RRSs per bed capacity, type of ownership, and geographic distribution.

\section{2) Data collection and in-hospital cardiopulmonary ar- rest rates}

Each hospital included in the study used varying types of cardiopulmonary resuscitation (CPR) reports based on their own definition of cardiopulmonary arrest, which differed by location, cardiac rhythm, and patient population. Broadly, these definitions encompass circumstances such as the cessation of cardiac mechanical activity, absence of a detectable pulse, unresponsiveness, and apnea [9]. In this study, in-hospital cardiopulmonary arrest cases included those in the general ward; cases where the arrest took place in the emergency department, operating room, or intensive care unit were excluded. The inhospital cardiopulmonary arrest rate was defined as the CPR rate/1,000 adult admissions. The statistics on the CPR rate of each hospital were gathered from CPR reports and statistics about adult admissions and discharges. We collected detailed data from each hospital from 2013 to 2015 regarding the cardiopulmonary arrest rate of patients at least 18 years of age in the general ward, the number of annual adult admissions, and whether a dedicated intensivist was present. Additionally, rapid response team characteristics were analyzed at the two institutions that had implemented RRSs.

To verify the clinical effect of RRSs, we selected a subset of hospitals among the 12 included institutions without RRSs. To minimize bias, we excluded the two hospitals with the highest and the lowest CPR rates and three hospitals that had not used electronic medical record (EMR) systems in 2013. Finally, seven hospitals were included in the group without an RRS. To identify the in-hospital cardiopulmonary arrest rate by hospital bed size, we categorized hospitals with more than 32,000 annual adult admissions (the median value among the 14 hospitals) as "high-volume hospitals" and those with less than 32,000 annual adult admissions as "medium-volume hospitals."

\section{3) Statistical analysis and ethical considerations}

The chi-square test for categorical dummy variables was performed to compare cardiopulmonary arrest rates prior to and after implementation of an RRS. We also investigated cardiopulmonary arrest rates in accordance with hospital characteristics of location, type of organization, and bed capacity. Statistical analysis was performed using SPSS for Windows version 18.0 (SPSS Inc., Chicago, IL, USA). Microsoft Excel 2010 (Microsoft, Redmond, WA, USA) was used to collect and summarize the data and generate graphs. A P-value less than 0.05 was regarded as statistically significant. Because we only collected statistical data regarding mortality and did not use personal information, the Institutional Review Board waived deliberation of this study (IRB No. 2016-12-006).

\section{Results}

\section{1) Hospital characteristics}

The characteristics of the 14 hospitals are presented Table 1. Twelve hospitals did not have an RRS, while two did have an RRS. In the group with an RRS, one hospital was a national university-affiliated institution, and the other was private and university-affiliated; both were located in provincial areas. Among the 12 hospitals without RRSs, four were in the capital area, and eight were situated in provincial areas. All hospitals were tertiary medical centers in urban settings. Of the locations 
Table 1. Characteristics of included hospitals

\begin{tabular}{|c|c|c|}
\hline Variable & $\begin{array}{l}\text { Hospital without } \\
\text { RRS }(n=12)\end{array}$ & $\begin{array}{l}\text { Hospital with } \\
\text { RRS }(n=2)\end{array}$ \\
\hline \multicolumn{3}{|l|}{ Region } \\
\hline Capital area & 4 & - \\
\hline Provincial area & 8 & 2 \\
\hline \multicolumn{3}{|l|}{ Type } \\
\hline Tertiary hospital & 12 & 2 \\
\hline General hospital & - & - \\
\hline \multicolumn{3}{|l|}{ Operating } \\
\hline National university-affiliated & 4 & 1 \\
\hline Private university-affiliated & 8 & 1 \\
\hline \multicolumn{3}{|l|}{ No. of admission (adult) } \\
\hline 20,000-29,999/yr & 6 & - \\
\hline $30,000-39,999 / y r$ & 3 & 2 \\
\hline 40,000-49,999/yr & 3 & - \\
\hline \multicolumn{3}{|l|}{ Capacity (bed) } \\
\hline $1,000-1,300$ & 2 & 1 \\
\hline 700-999 & 10 & 1 \\
\hline \multicolumn{3}{|l|}{ Full-time intensivist ${ }^{\mathrm{a}}$} \\
\hline Yes & 9 & 2 \\
\hline No & 3 & - \\
\hline \multicolumn{3}{|l|}{$\begin{array}{l}\text { Presence of training program for } \\
\text { CPR or BLS }\end{array}$} \\
\hline Yes & 12 & 2 \\
\hline No & 0 & 0 \\
\hline \multicolumn{3}{|l|}{$\begin{array}{l}\text { Presence of department of } \\
\text { quality improvement }\end{array}$} \\
\hline Yes & 12 & 2 \\
\hline No & 0 & 0 \\
\hline
\end{tabular}

RRS: rapid response system; CPR: cardiopulmonary resuscitation; BLS: basic life support.

${ }^{a}$ On July 1, 2015.

without an RRS, four hospitals were national universityaffiliated and eight were private university-affiliated. In terms of the annual number of adult admissions, half of the hospitals without an RRS admitted over 30,000 patients per year, and both hospitals with an RRS admitted 30,000-39,999 patients annually. Three hospitals without an RRS did not have full-time intensivists on staff as of July 2015. In addition, all 14 hospitals offered CPR or basic life support training programs for healthcare providers and implemented departments of quality improve-

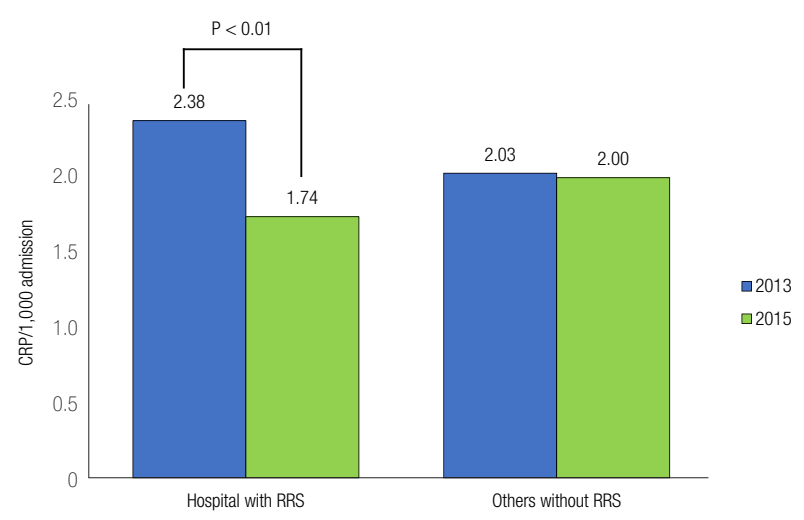

Figure 2. A comparison of cardiopulmonary arrest rates between hospitals with RRS and without RRS. CPR: cardiopulmonary resuscitation; RRS: rapid response system.

ment. Detailed characteristics of the two hospitals with an RRS are presented in Supplementary Table 1.

\section{2) Comparison of in-hospital cardiopulmonary arrest rates}

We compared the cardiopulmonary arrest rates between the hospitals with an RRS and those without it (Figure 2). Hospitals with an RRS showed a statistically significant reduction in CPR rate between 2013 and 2015 (odds ratio $[\mathrm{OR}], 0.731 ; 95 \%$ confidence interval $[\mathrm{CI}], 0.577$ to $0.927 ; \mathrm{P}=0.009)$. We analyzed the monthly $\mathrm{CPR}$ rate of the two hospitals with an RRS (Figure 3), which demonstrated a gradual reduction in CPR rate. The incidence of CPR in 2013 and 2015 was similar in hospitals without an RRS (OR, $0.988 ; 95 \% \mathrm{CI}, 0.868$ to $1.124 ; \mathrm{P}=$ 0.854). Table 2 displays the annual CPR rates and yearly adult admissions statistics in each of the two groups; the number of CPR episodes declined from 161 cases to 119 cases after the implementation of an RRS.

\section{3) Prevalence of in-hospital cardiopulmonary arrest}

We verified the in-hospital cardiopulmonary arrest rates of hospitals based on affiliation (national vs. private), location (capital vs. provincial), and volume (highvolume vs. medium-volume). We additionally excluded three hospitals that did not use an EMR in 2013 to avoid selection bias. Therefore, all included hospitals utilized an EMR during the period of interest (Table 3). 


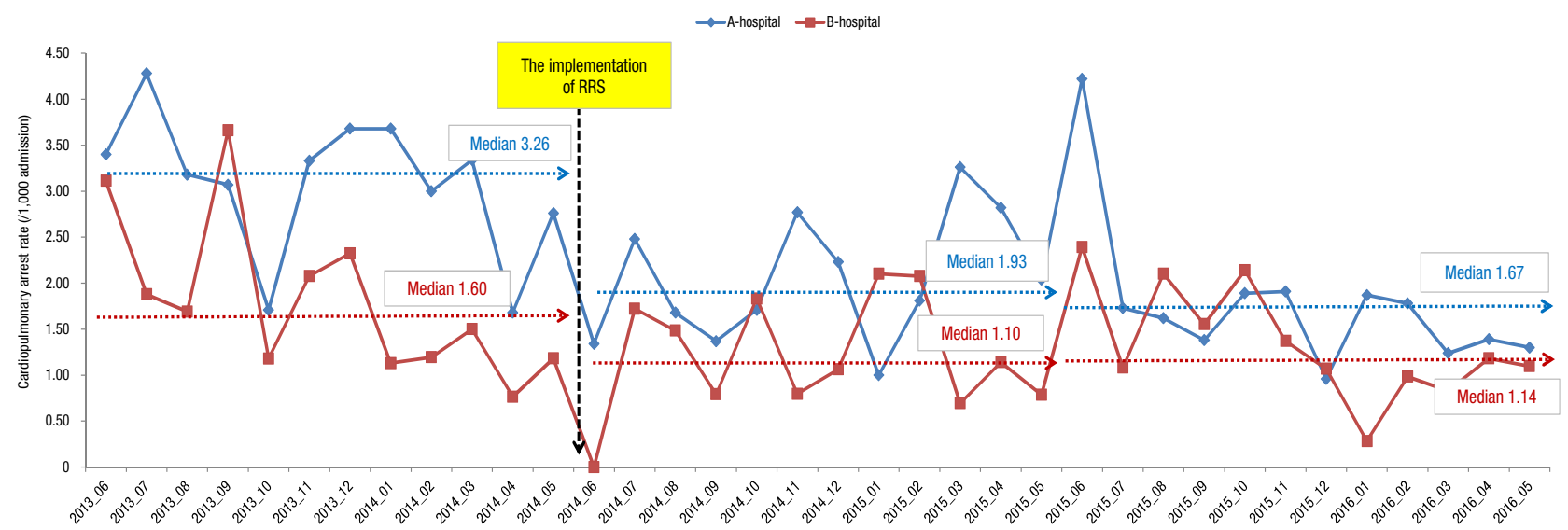

Figure 3. The monthly cardiopulmonary arrest rate of each hospital with an RRS. RRS: rapid response system.

Table 2. Annual CPR number and annual adult admission number of two groups

\begin{tabular}{ccc}
\hline Variable & $\begin{array}{c}\text { 2 Hospitals } \\
\text { with RRS }\end{array}$ & $\begin{array}{c}7 \text { Hospitals } \\
\text { without RRS }\end{array}$ \\
\hline No. of CPR & 161 & 454 \\
\hline In 2013 & 119 & 471 \\
\hline In 2015 & & \\
No. of admissions (adult) & 67,770 & 224,105 \\
\hline In 2013 & 68,438 & 235,319 \\
\hline In 2015 & & \\
\hline CPR rate (per 1,000 admission) & 2.38 & 2.03 \\
\hline In 2013 & 1.74 & 2.00 \\
\hline In 2015 & & \\
\hline
\end{tabular}

CPR: cardiopulmonary resuscitation; RRS: rapid response system.

\section{(1) National university-affiliated hospitals versus private university-affiliated hospitals}

From 2013 to 2015, the in-hospital cardiopulmonary arrest rate of the five national university-affiliated hospitals decreased from 2.29 to 1.92 , and that of the nine private university-affiliated hospitals increased from 2.04 to 2.40. National university-affiliated hospitals had a significantly lower OR than private hospitals in 2015 (1.92 vs. 2.40, respectively; OR, $0.800 ; 95 \% \mathrm{CI}, 0.702$ to 0.912 ; $\mathrm{P}=0.001)$ (Figure 4A).

\section{(2) Capital area versus provincial area}

Four hospitals represented the capital area and demonstrated a significant decrease in cardiopulmonary arrest rate (1.93 to 1.53$)$ over 2 years. However, in the remaining 10 hospitals, the cardiopulmonary arrest rate increased from 2.19 to 2.51 . In 2015 , the capital area showed a significantly lower cardiopulmonary arrest rate compared to hospitals in provincial areas (1.53 vs. 2.51, respectively; OR, 0.609 ; $95 \% \mathrm{CI}, 0.523$ to 0.709 ; $\mathrm{P}<$ 0.001) (Figure 4B).

\section{(3) High-volume hospitals versus medium-volume hospitals}

High-volume hospitals showed fewer in-hospital cardiopulmonary arrests compared with medium-volume hospitals in 2013 (1.76 vs. 2.63, respectively; OR, 0.667; $95 \%$ CI, 0.577 to $0.772 ; \mathrm{P}<0.001)$ and in 2015 (1.55 vs. 3.20 , respectively; OR, 0.485 ; $95 \% \mathrm{CI}, 0.428$ to 0.550 ; $\mathrm{P}$ $<0.001$ ) (Figure 4C).

\section{Discussion}

Prior studies have suggested that in-hospital mortality is not an appropriate indicator of hospital service or quality given that patient populations vary widely among hospitals, and a comparison of mortality without any correction for selection bias could skew such analyses [10-12]. Nonetheless, the number of in-hospital cardiopulmonary arrests can be reduced with proper interventions; this is a hot topic of interest for administrators and 
Table 3. Categorical data for in-hospital cardiopulmonary arrest rate at all 14 hospitals

\begin{tabular}{|c|c|c|c|c|}
\hline & $\begin{array}{l}2013 \text { Cardiopulmonary } \\
\text { arrest }(n=11)\end{array}$ & $\mathrm{OR}(95 \% \mathrm{Cl})$ & $\begin{array}{l}2015 \text { Cardiopulmonary } \\
\text { arrest }(n=14)\end{array}$ & $\mathrm{OR}(95 \% \mathrm{Cl})$ \\
\hline Ownership & & $1.124(0.957-1.318)$ & & $0.800(0.702-0.912)$ \\
\hline National & 2.29 & & 1.92 & \\
\hline Private & 2.04 & & 2.40 & \\
\hline Location & & $0.882(0.757-1.028)$ & & $0.609(0.523-0.709)$ \\
\hline Capital area & 1.93 & & 1.53 & \\
\hline Provincial area & 2.19 & & 2.51 & \\
\hline Annual admission number & & $0.667(0.577-0.772)^{*}$ & & $0.485(0.428-0.550)$ \\
\hline$>32,000$ & 1.76 & & 1.55 & \\
\hline$<32,000$ & 2.63 & & 3.20 & \\
\hline
\end{tabular}

OR: odds ratio; $\mathrm{Cl}$ : confidence interval. ${ }^{*} \mathrm{P}<0.05$.

(A)

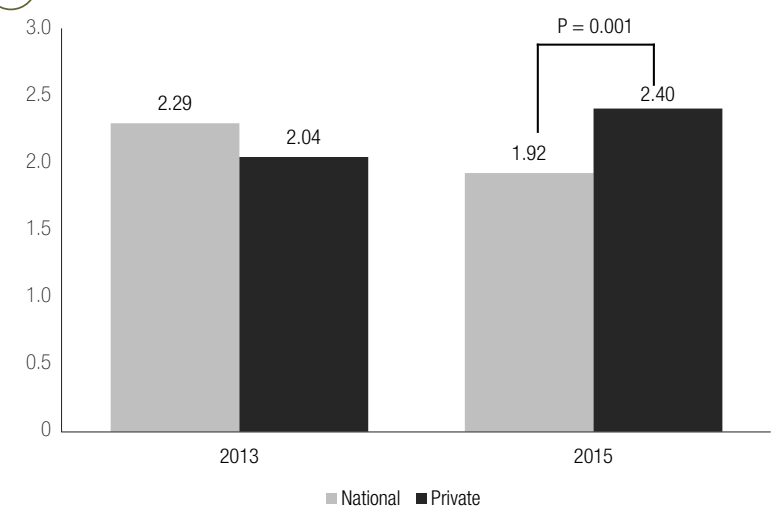

(C)

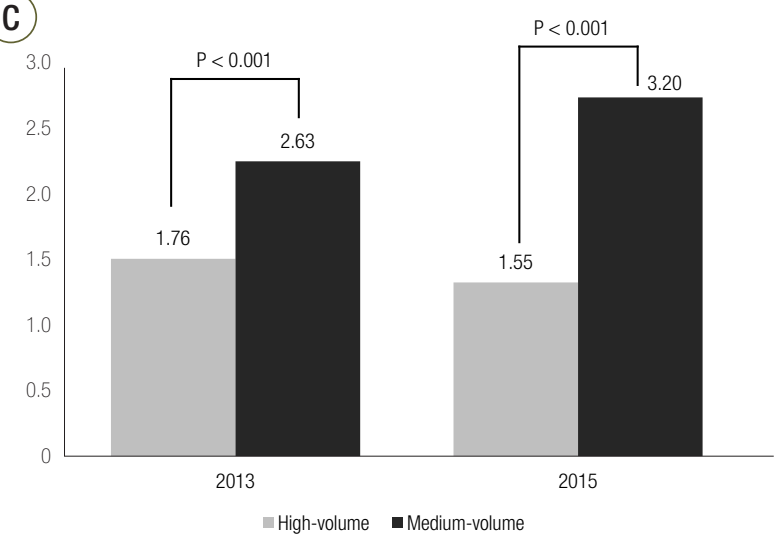

healthcare policy authorities. Various ways to prevent cardiopulmonary arrest have been introduced over the past few decades. They include the introduction of an EMR, use of Early Warning Score systems, creation of intermediate care units, and implementation of an RRS
(B)

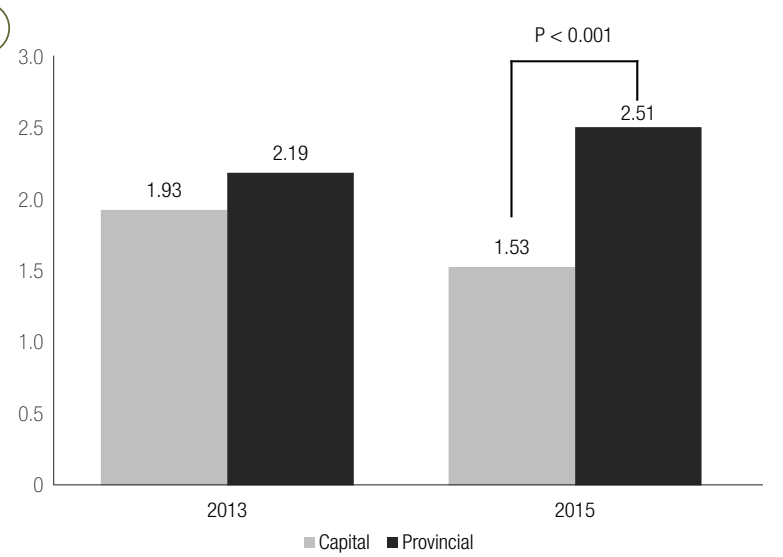

Figure 4. A comparison of in-hospital cardiac arrest rate according to hospital characteristics. (A) National university-affiliated hospitals versus private university-affiliated hospitals, (B) capital area versus provincial area, (C) high-volume hospitals versus medium-volume hospitals.

\section{$[1,13,14]$}

RRSs have been widely adopted around the world over the past two decades and effectively reduce in-hospital cardiopulmonary arrests [13-18]. RRSs can diminish inhospital cardiopulmonary arrests and improve patient 
safety by initiating earlier treatment attempts; impacting do-not-attempt-resuscitation decisions; monitoring atrisk patients; increasing levels of care; correcting wrong diagnoses; and requiring specialist consultations [19].

Despite these benefits, there have been barriers against the successful implementation of RRSs. Practically, there is a lack of specialists for RRS implementation and scarce financial support by hospitals. Moreover, the absence of government policy about RRSs is an important issue to be addressed. It is worth mentioning that some multi-center trials on RRSs have been conducted in other countries, and their results deserve attention despite being obtained in different healthcare systems and environments $[7,16,17]$.

In this survey, the cardiopulmonary arrest rate of the 14 participating tertiary hospitals ranged from 1.02 to 7.1/1,000 adult admissions for the study period. Five hospitals without an RRS showed a low incidence of cardiopulmonary arrest ( $<2.0 / 1,000$ admissions), whereas three hospitals without an RRS reported a higher incidence of cardiopulmonary arrest (>3.0/1,000 admissions). These variations in cardiopulmonary arrest rates might result from differences in hidden curricula and workforces, but this is beyond the scope of this study.

Interestingly, this pilot analysis demonstrated that two hospitals with an operating RRS achieved a statistically significant reduction of in-hospital cardiopulmonary arrests between 2013 and 2015, although hospital A experienced an abrupt rise in the CPR rate in July 2015 due to an outbreak of Middle East respiratory syndrome.

Regardless of the presence of RRSs, we found different cardiopulmonary arrest rates based on hospital characteristics. National university-affiliated hospitals showed relatively fewer cardiopulmonary arrests than private hospitals in 2015. High-volume hospitals also demonstrated a lower cardiopulmonary arrest rate than mediumvolume hospitals. This tendency was presumed to be due to the strong medical and human resources available in national university-affiliated hospitals and high-volume hospitals. While all high-volume hospitals had dedicated intensivists, only $57 \%$ (four of seven hospitals) of me- dium-volume hospitals had them. None of the mediumvolume hospitals had an RRS. Furthermore, three of five national university-affiliated hospitals $(60 \%)$ were highvolume hospitals, whereas four of nine private hospitals (44\%) were high-volume hospitals, which could explain the differences between cardiopulmonary arrest at national university-affiliated hospitals and private hospitals (Supplementary Table 2). However, these results should be interpreted with caution because this study was not a comprehensive investigation, but instead only compared the structural characteristics of a few hospitals. All included hospitals were tertiary hospitals and were accredited by Ministry of Health and Welfare. Accordingly, it can be assumed that the levels of care were above standard.

In 2015, hospitals in the capital area demonstrated a lower cardiopulmonary arrest rate than those in provincial areas. Two of these were high-volume hospitals, but that difference does not fully establish an inference related with patient volume. Although we could not entirely rule out a selection bias in our sampling methods, this result might suggest that the discrepancy in cardiopulmonary arrest rate is due to a local gap in medical services as well as the severity of patient illnesses and comorbidities.

This is the first multicenter survey on the impacts of RRSs in Korea. To avoid selection bias, we made efforts to study hospitals with similar characteristics. First, we corrected combined probability sampling with nonprobability sampling. Second, we excluded data from hospitals that recorded the highest and lowest cardiopulmonary arrest rates and from the period when EMRs were not used. However, there are some limitations to this investigation. This study was a retrospective analysis that only included data from 14 of the 43 tertiary hospitals, which could have introduced a selection bias. Regarding the data, we collected admission statistics from each hospital and did not review individual medical records. Each hospital had a different system of reporting CPR rates, including standards to fulfill CPR reports and responsible parties. Hence, it is possible that some data are not apparent from the accessible CPR cases. Nevertheless, the aim of this research was to determine the incidence of cardiopul- 
monary arrest, not to analyze its causes. It was also not intended to be a comparative analysis that reflected various variables and characteristics of each hospital. Finally, this study was designed as a before and after study, and it is challenging to conduct randomized controlled trials in this manner.

The implementation of an RRS in two tertiary hospitals reduced the incidence of cardiopulmonary arrest over 3 years. Although we cannot conclude that RRSs are the only method that can be used to reduce cardiopulmonary arrest, they might be a practical option to use to achieve this goal. The discrepancies in cardiopulmonary arrest rate submitted for this survey suggest that a nationwide survey on cardiopulmonary arrest and the effect of RRSs is necessary. Promoting patient safety and healthcare quality is within the public domain. Therefore, hospital administrators should take an interest in encouraging and supporting the implementation of RRSs.

\section{Acknowledgments}

We are grateful to the rapid response teams at Chungnam National University Hospital and Ulsan University Hospital for their key role in this work.

Author contributions:

substantial contributions to conception and design, acquisition of data or analysis and interpretation of data: all authors; drafting the article or revising it critically for important intellectual content: YHP, JJA, BJK, GHH, SWS, JHK, JYM; final approval of the version to be published: all authors.

\section{ORCID}

Yeonhee Park

Byung Ju Kang

Young Seok Lee

Sang-Ook Ha

Dong-Hyun Lee

Jae Young Moon http://orcid.org/0000-0001-9537-1530

http://orcid.org/0000-0002-1396-7398

http://orcid.org/0000-0002-0144-2033

http://orcid.org/0000-0002-2651-1023

http://orcid.org/0000-0001-6253-3396

http://orcid.org/0000-0001-8724-6289

\section{Supplementary Materials}

The online-only Supplement data are available with this article online: https://doi.org/10.4266/kjccm.2017.00024.

\section{References}

1. Morrison LJ, Neumar RW, Zimmerman JL, Link MS, Newby LK, McMullan PW Jr, et al. Strategies for improving survival after in-hospital cardiac arrest in the United States: 2013 consensus recommendations--a consensus statement from the American Heart Association. Circulation 2013;127:1538-63.

2. Skrifvars MB, Nurmi J, Ikola K, Saarinen K, Castrén M. Reduced survival following resuscitation in patients with documented clinically abnormal observations prior to in-hospital cardiac arrest. Resuscitation 2006;70:215-22.

3. Sandroni C, Nolan J, Cavallaro F, Antonelli M. Inhospital cardiac arrest: incidence, prognosis and possible measures to improve survival. Intensive Care Med 2007;33:237-45.

4. Hillman KM, Bristow PJ, Chey T, Daffurn K, Jacques T, Norman SL, et al. Antecedents to hospital deaths. Intern Med J 2001;31:343-8.

5. Buist MD, Moore GE, Bernard SA, Waxman BP, Anderson JN, Nguyen TV. Effects of a medical emergency team on reduction of incidence of and mortality from unexpected cardiac arrests in hospital: preliminary study. BMJ 2002;324:387-90.

6. Schein RM, Hazday N, Pena M, Ruben BH, Sprung CL. Clinical antecedents to in-hospital cardiopulmonary arrest. Chest 1990;98:1388-92.

7. Chen J, Ou L, Hillman K, Flabouris A, Bellomo R, Hollis SJ, et al. The impact of implementing a rapid response system: a comparison of cardiopulmonary arrests and mortality among four teaching hospitals in Australia. Resuscitation 2014;85:1275-81.

8. Chen J, Ou L, Hillman KM, Flabouris A, Bellomo R, Hollis SJ, et al. Cardiopulmonary arrest and mortal- 
ity trends, and their association with rapid response system expansion. Med J Aust 2014;201:167-70.

9. Jacobs I, Nadkarni V, Bahr J, Berg RA, Billi JE, Bossaert L, et al. Cardiac arrest and cardiopulmonary resuscitation outcome reports: update and simplification of the Utstein templates for resuscitation registries. A statement for healthcare professionals from a task force of the international liaison committee on resuscitation (American Heart Association, European Resuscitation Council, Australian Resuscitation Council, New Zealand Resuscitation Council, Heart and Stroke Foundation of Canada, InterAmerican Heart Foundation, Resuscitation Council of Southern Africa). Resuscitation 2004;63:233-49.

10. Thomas JW, Hofer TP. Accuracy of risk-adjusted mortality rate as a measure of hospital quality of care. Med Care 1999;37:83-92.

11. Hofer TP, Hayward RA. Identifying poor-quality hospitals: can hospital mortality rates detect quality problems for medical diagnoses? Med Care 1996;34:737-53.

12. Zalkind DL, Eastaugh SR. Mortality rates as an indicator of hospital quality. J Healthc Manag 1997;42:3-15.

13. DeVita MA, Braithwaite RS, Mahidhara R, Stuart S,
Foraida M, Simmons RL, et al. Use of medical emergency team responses to reduce hospital cardiopulmonary arrests. Qual Saf Health Care 2004;13:251-4.

14. Goldhill DR, Worthington L, Mulcahy A, Tarling M, Sumner A. The patient-at-risk team: identifying and managing seriously ill ward patients. Anaesthesia 1999;54:853-60.

15. Winters BD, Weaver SJ, Pfoh ER, Yang T, Pham JC, Dy SM. Rapid-response systems as a patient safety strategy: a systematic review. Ann Intern Med 2013;158(5 Pt 2):417-25.

16. Maharaj R, Raffaele I, Wendon J. Rapid response systems: a systematic review and meta-analysis. Crit Care 2015;19:254.

17. Chan PS, Jain R, Nallmothu BK, Berg RA, Sasson C. Rapid response teams: a systematic review and meta-analysis. Arch Intern Med 2010;170:18-26.

18. Bellomo R, Goldsmith D, Uchino S, Buckmaster J, Hart GK, Opdam H, et al. A prospective before-andafter trial of a medical emergency team. Med J Aust 2003;179:283-7.

19. Direkze S, Jain S. Time to intervene? Lessons from the NCEPOD cardiopulmonary resuscitation report 2012. Br J Hosp Med (Lond) 2012;73:585-7. 